\title{
Ethnic Disparities in Blood Pressure Manage- ment in Patients With Hypertension After the Introduction of Pay for Performance
}

\author{
Christopher Millett, PbD, FFPH ${ }^{1,2}$ \\ Jeremy Gray, MBBS, MRCP ${ }^{2}$ \\ Alex Bottle, MSc, PbD ${ }^{1}$ \\ Azeem Majeed, MD, FRCGP' \\ 'Department of Primary Care \& Social \\ Medicine, Imperial College Faculty of \\ Medicine, London, England \\ ${ }^{2}$ Wandsworth Primary Care Research \\ Centre, Wandsworth Primary Care Trust, \\ London, England
}

\begin{abstract}
PURPOSE Little is known about the impact of pay-for-performance incentives on health care disparities. We examined ethnic disparities in the management of hypertension among patients with and without cardiovascular comorbidities after the implementation of a major pay-for-performance incentive scheme in UK primary care.
\end{abstract}

METHODS We undertook a population-based, cross-sectional survey of medication prescriptions and blood pressure control among patients with hypertension using electronic medical records from 16 family practices in southwest London.

RESULTS Black patients with hypertension were significantly less likely to achieve an established treatment target for blood pressure control than white or South Asian patients (adjusted odds ratio, 0.86; 95\% confidence interval, 0.74-0.99). The prevalence of cardiovascular comorbidities was higher among South Asian patients with hypertension than among their white or black counterparts (41.3\% vs $28.5 \%$ vs $28.8 \%$ ). The presence of 2 or more cardiovascular comorbidities was associated with significantly improved blood pressure control among white patients but not among black or South Asian patients (mean systolic blood pressure, $-9.4 \mathrm{~mm} \mathrm{Hg},-0.6 \mathrm{~mm} \mathrm{Hg}$, and $-1.8 \mathrm{~mm} \mathrm{Hg}$, respectively). South Asian patients with poorly controlled hypertension were prescribed fewer antihypertensive medications than their black or white peers (adjusted odds ratio, 0.66; 95\% confidence interval, 0.46-0.96).

CONCLUSIONS Ethnic disparities in the management of hypertension have persisted in the United Kingdom despite major investment in quality improvement initiatives, including pay for performance. These disparities are particularly marked among patients with multiple cardiovascular conditions.

Ann Fam Med 2008;6:490-496. DOI: 10.1370/afm.907.

\section{INTRODUCTION}

$\mathrm{H}$ ypertension is an important determinant of ethnic disparities in health. The prevalence of hypertension is considerably higher among black African and Caribbean individuals than among white individuals, ${ }^{1,2}$ and the hypertension-associated risk of cardiovascular disease may be accentuated in South Asian individuals. ${ }^{3}$ High-quality management of hypertension is especially important in black and South Asian groups as they are more likely than their white counterparts to have coexisting cardiovascular comorbidities, such as diabetes. ${ }^{4,5}$

Over the past decade, the UK government has implemented an ambitious quality improvement agenda, a key objective of which is to reduce disparities in the quality of chronic disease management. ${ }^{6}$ This agenda includes a series of National Service Frameworks for chronic conditions such as coronary heart disease, stroke, and diabetes, and the implementation of the Quality and Outcomes Framework as part of a new family 
practitioner contract in 2004. Described as the boldest attempt to link pay to performance in any health care system, ${ }^{7}$ the framework places considerable emphasis on improving the quality of care for individuals with cardiovascular disease.

Initiatives to improve the quality of health care, including pay-for-performance incentives, may produce unintended and undesirable consequences. For example, pay-for-performance incentives may worsen health disparities if quality improves more slowly among professionals serving ethnically diverse populations with a high disease burden. ${ }^{8}$

In this study, we examined ethnic disparities in the management of hypertension among individuals with and without cardiovascular comorbidities after the introduction of a major pay-for-performance program in the United Kingdom. Ethnic disparities in the quality of health care are a persistent feature in the country, despite a lack of financial barriers to accessing health care, universal health coverage, and numerous initiatives to reduce disparities. ${ }^{9}$ A clear conceptual framework for how disparities in quality of care and health care outcomes may arise is given in an overview by Arleen Brown and colleagues. ${ }^{10}$ Differences in the quality of care may arise through a number of mechanisms, including financial barriers, education, healthseeking behavior, and access to health services. In our study, we examined the end result of these mechanisms in a health care system offering universal health coverage to all segments of the population.

\section{METHODS}

\section{Pay for Performance in UK Primary Care}

Pay for performance was introduced in UK primary care as part of the new General Practitioner contract in April 2004. About one-quarter of general practice income is now derived by achieving quality targets in managing chronic diseases such as diabetes and coronary heart disease, and risk factors such as hypertension, through the Quality and Outcomes Framework. The Framework consists of 1,000 points, which cover clinical care, practice organization, and patient experience.

Hypertension is 1 of 19 areas within the clinical domain of the Quality and Outcomes Framework. Of the 83 points available for hypertension, 6 are assigned to the production of a registry of hypertensive patients, 20 are allocated to measurement of blood pressure in the previous 9 months (40\%-90\% of patients must achieve the target to obtain payment), and 57 are allocated to achieving a treatment target blood pressure of $150 / 90 \mathrm{~mm} \mathrm{Hg}$ or less (40\%-70\% of patients must achieve the target to obtain payment).
Blood pressure measurement in UK primary care is undertaken by family practitioners or practice nurses who enter values into electronic medical records.

\section{Study Sample}

The study was conducted in Wandsworth, southwest London, where residents are younger, more deprived, and more culturally diverse than is typical in England as a whole. ${ }^{11,12}$ The area covered by the study contained 16 primary care practices with a total registered population of 120,843 patients. Data for the present study were collected from these practices between November 2005 and January 2006. The study was approved by the Wandsworth Research Ethics Committee.

We identified all patients with essential hypertension using diagnostic Read codes in computerized family practice records. Read codes are the clinical classification system used in primary care in the United Kingdom. ${ }^{13}$ We then extracted clinical information, including information about cardiovascular comorbidities (coronary heart disease, heart failure, stroke, diabetes, and chronic renal failure), blood pressure values (the most recent reading), and currently prescribed antihypertensive medications. Patients reported their ethnic group to the family practices on the basis of the classifications that map to the 2001 UK census $^{12}{ }^{12}$ either at registration or during a consultation at the practice. We categorized ethnicity into 3 groups (white, black, South Asian) for our analyses because numbers of patients in individual ethnic subgroups were small. A neighborhood socioeconomic status (SES) score was assigned to individual patients based on their postal code using the Index of Multiple Deprivation (IMD) 2004. ${ }^{11}$ This index is the most commonly used method of measuring neighborhood SES in the United Kingdom and is compiled from a variety of sources, including the 2001 UK census and unemployment and social security benefits records. Higher scores on this index indicate greater deprivation.

\section{Data Analyses}

For each ethnic group, we calculated mean systolic and diastolic blood pressure, and percentage of patients achieving an established treatment target (blood pressure $<140 / 90 \mathrm{~mm} \mathrm{Hg}$ ) among patients with 0,1 , and 2 or more cardiovascular comorbidities. We calculated the number and type of antihypertensive medications prescribed in each group. Regression analysis was undertaken to examine associations between ethnicity, number of comorbidities, and blood pressure control, with age, sex, neighborhood SES score, and treatment (number of antihypertensive medications prescribed) as the independent variables. We used robust standard errors to take into account 
clustering of patients within general practices. ${ }^{14}$ Statistical analyses were performed using version 9.1 of Stata (Stata Corp, College Station, Texas).

\section{RESULTS}

We identified 9,044 patients with essential hypertension in the 16 participating primary care practices. A total of 168 (1.9\%) were subsequently excluded because they had an incomplete or irregular blood pressure reading. Ethnicity was recorded for $94.8 \%$ of the remaining 8,876 patients; $45.4 \%$ were white British, 26.7\% were black (Caribbean, $16.5 \%$; African, 10.2\%), 5.6\% were South Asian (Indian, 3.5\% ; Pakistani, 1.7\%; Bangladeshi, 0.4\%), and $17.1 \%$ belonged to other ethnic groups. On average, black patients lived in more deprived neighborhoods than their white or South Asian counterparts (mean neighborhood SES scores, 26.4, 22.2, and 20.9, respectively).

Women comprised a larger proportion of hypertensive patients without cardiovascular comorbidities than men $(59.4 \%$ vs $40.6 \%$ ), but this pattern was reversed among patients with 2 or more comorbidities (39.5\% vs $60.5 \%$ ) (Table 1). Some $15.6 \%$ of the sample had diabetes, $12.3 \%$ had coronary heart disease, $3.1 \%$ had experienced a stroke, $2.7 \%$ had heart failure, and $1.5 \%$ had chronic renal failure. South Asian patients were more likely to have comorbidities than white or black patients ( $41.3 \%$ vs $28.5 \%$ vs $28.8 \%$ ). Hypertensive black and South Asian patients with and without comorbidities were considerably younger than their white counterparts (Table 1).

The percentage of patients achieving the quality target for blood pressure measurement in the family practitioner contract (measurement of blood pressure within the past 9 months) was similar in the white, black, of deprivation. and South Asian groups at 94.5\%, 94.9\%, and 95.3\%, respectively. Black hypertensive patients had significantly higher mean blood pressure values and were significantly less likely to meet the treatment target for blood pressure control $(<140 / 90 \mathrm{~mm} \mathrm{Hg})$ than white patients (adjusted odds ratio [AOR], 0.86; 95\% confidence interval [CI], 0.74-0.99) or South Asian patients.

Hypertensive patients with cardiovascular comor-

\begin{tabular}{|c|c|c|c|c|}
\hline Characteristic & $\begin{array}{c}0 \\
\text { Comorbidities } \\
(n=6,329)\end{array}$ & $\begin{array}{c}1 \\
\text { Comorbidity } \\
(n=2,056)\end{array}$ & $\begin{array}{c}\geq 2 \\
\text { Comorbidities } \\
(n=491)\end{array}$ & $\begin{array}{c}\text { Total } \\
(\mathrm{N}=\mathbf{8}, 876)\end{array}$ \\
\hline Sex, \% male & 40.6 & 50.7 & 60.5 & 44.1 \\
\hline \multicolumn{5}{|l|}{ Age, mean, years } \\
\hline White & 64.6 & 70.7 & 76.0 & 66.6 \\
\hline Black & 58.1 & 64.9 & 70.7 & 60.4 \\
\hline South Asian & 59.2 & 63.7 & 68.6 & 61.7 \\
\hline \multicolumn{5}{|l|}{ Ethnicity, \% } \\
\hline White & 71.5 & 23.4 & 5.2 & - \\
\hline Black & 71.2 & 23.6 & 5.2 & - \\
\hline South Asian & 58.7 & 28.7 & 12.6 & - \\
\hline \multicolumn{5}{|l|}{ BMI, mean, kg/m² } \\
\hline White & 27.6 & 28.7 & 29.2 & 27.9 \\
\hline Black & 29.6 & 29.6 & 29.1 & 29.6 \\
\hline South Asian & 27.5 & 27.7 & 26.8 & 27.5 \\
\hline \multicolumn{5}{|l|}{ Smokers, \% } \\
\hline White & 23.6 & 21.6 & 18.3 & 22.8 \\
\hline Black & 12.3 & 9.5 & 10.6 & 11.6 \\
\hline South Asian & 8.7 & 6.3 & 8.1 & 7.9 \\
\hline $\begin{array}{l}\text { Neighborhood } \\
\text { SES score, } \\
\text { mean }^{\text {a }}\end{array}$ & 23.1 & 23.3 & 23.9 & 23.2 \\
\hline
\end{tabular}

$\mathrm{BMI}=$ body mass index; $\mathrm{SES}=$ socioeconomic status.

'Based on the Index of Multiple Deprivation 2004; the higher the score, the greater the neighborhood's level

\section{Table 2. Blood Pressure Control by Number of Cardiovascular} Comorbidities and Ethnicity

\begin{tabular}{|c|c|c|c|}
\hline Measure and Group & $\begin{array}{c}0 \\
\text { Comorbidities }\end{array}$ & $\begin{array}{c}1 \\
\text { Comorbidity }\end{array}$ & $\begin{array}{c}\geq 2 \\
\text { Comorbidities }\end{array}$ \\
\hline \multicolumn{4}{|c|}{ Systolic BP, mean (SE), mm Hg } \\
\hline White & $140.7(0.3)$ & $138.0(0.6)$ & $133.3(1.5)$ \\
\hline Black & $141.8(0.4)$ & $140.2(0.7)$ & $142.2(1.6)$ \\
\hline South Asian & $139.1(1.0)$ & $136.4(1.5)$ & $140.3(2.8)$ \\
\hline \multicolumn{4}{|c|}{ Diastolic BP, mean (SE), mm Hg } \\
\hline White & $81.5(0.2)$ & $77.2(0.3)$ & $72.5(0.7)$ \\
\hline Black & $84.4(0.3)$ & $79.3(0.5)$ & $76.4(1.0)$ \\
\hline South Asian & $80.9(0.6)$ & $78.8(1.0)$ & $74.0(1.7)$ \\
\hline \multicolumn{4}{|l|}{$\mathrm{BP}<140 / 90 \mathrm{~mm} \mathrm{Hg}, \%$} \\
\hline White & 42.5 & 52.6 & 56.7 \\
\hline Black & 42.0 & 47.4 & 46.3 \\
\hline South Asian & 44.8 & 57.0 & 54.8 \\
\hline
\end{tabular}




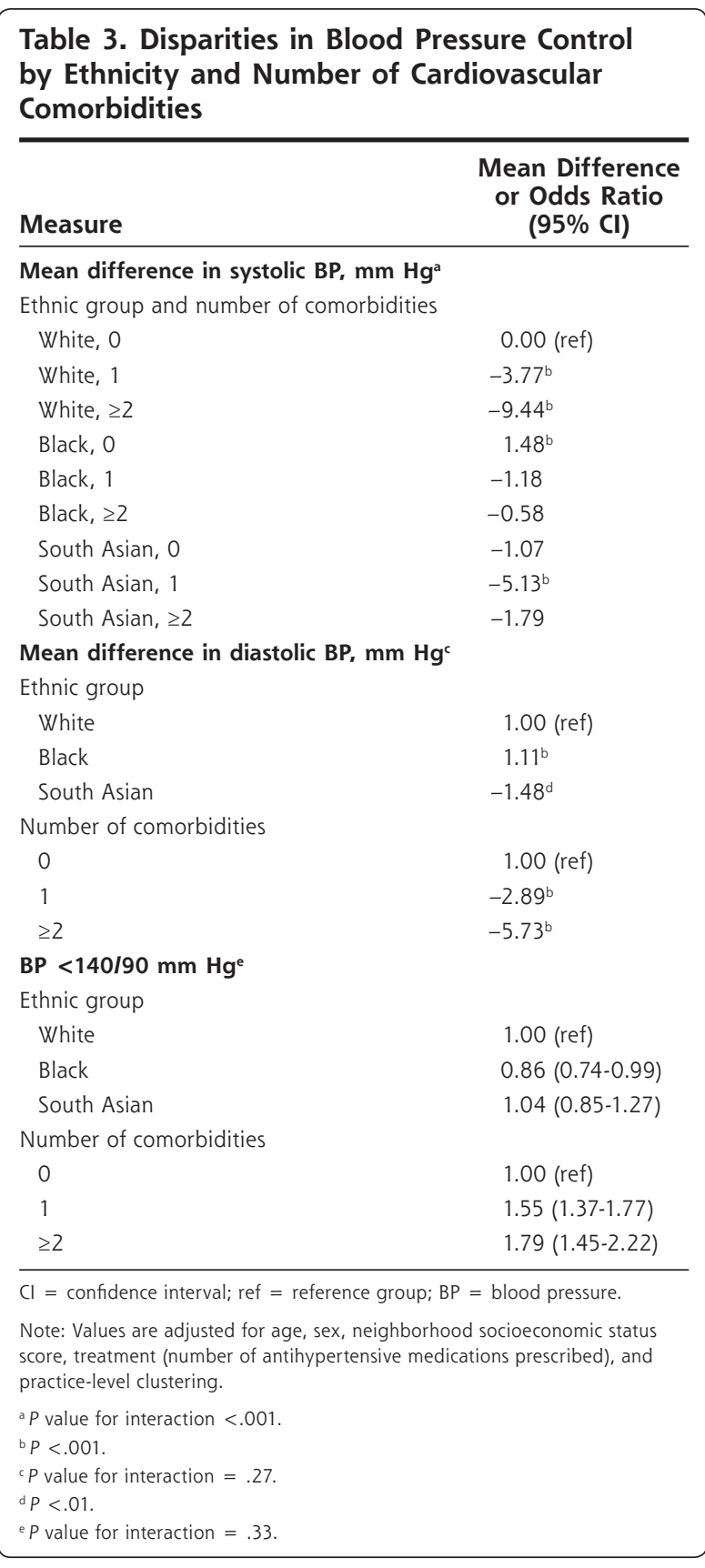

bidities were significantly more likely to meet the treatment target for blood pressure control than their peers without cardiovascular comorbidities (AOR, 1.79; 95\% CI, 1.45-2.22). Associations between the presence of cardiovascular comorbidities and mean systolic blood pressure differed significantly between ethnic groups $(P=.001)$ (Tables 2 and 3$)$. When white patients without comorbidity were the reference group, mean systolic blood pressure was significantly lower in white $(-3.8 \mathrm{~mm} \mathrm{Hg})$ and South Asian (-5.1 $\mathrm{mm} \mathrm{Hg}$ ) patients with 1 cardiovascular comorbidity, and in white patients with 2 or more comorbidities
(-9.4 mm Hg). In contrast, this improved control was not evident in black $(-0.6 \mathrm{~mm} \mathrm{Hg})$ or South Asian patients $(-1.8 \mathrm{~mm} \mathrm{Hg})$ with 2 more comorbidities. No interaction effect was evident for diastolic blood pressure $(P=.27)$ or achievement of the treatment target for hypertension $(P=.33)$.

National clinical guidelines in England ${ }^{15}$ advocate use of an angiotensin-converting enzyme inhibitor, $\beta$-blocker, calcium antagonist, and diuretic $(\mathrm{AB} / \mathrm{CD})$ treatment algorithm, where the CD combination is recommended for optimal management of hypertension in all black patients and older white patients. We found broad adherence to this guidance in black patients, who were more commonly prescribed calcium antagonists, diuretics, or both than were white and South Asian patients (Table 4).

Overall, 13\% of patients with poorly controlled hypertension (>140/90 $\mathrm{mm} \mathrm{Hg}$ ) were prescribed 3 or more antihypertensive medications, but values differed across ethnic groups (Table 4). The percentage was significantly lower in South Asian patients (AOR, 0.66; 95\% CI, 0.46-0.96) compared with white and black patients.

One in 5 patients $(19.4 \%)$ with poorly controlled hypertension and a single cardiovascular comorbidity were prescribed 3 or more antihypertensive medications; the value was $27.2 \%$ in black, $13.1 \%$ in South Asian, and $21.3 \%$ in white patients (Table 5). One in 3 patients $(34.4 \%)$ with poorly controlled hypertension and multiple comorbidities were prescribed 3 or more antihypertensive medications; the value was lower in white patients $(31.1 \%)$ and South Asian patients (28.6\%) when compared with black patients (40.9\%).

The relatively greater use of calcium antagonists among black patients persisted when patients were stratified according to number of cardiovascular comorbidities (Table 6). Some $61 \%$ of black patients with a single comorbidity were prescribed one of these agents, compared with $41 \%$ of similar white patients and $36 \%$ of similar South Asian patients. Likewise, $68 \%$ of black patients with multiple comorbidities were prescribed a calcium antagonist, relative to $43 \%$ of their white counterparts and $61 \%$ of their South Asian counterparts.

\section{DISCUSSION}

Ethnic disparities in the management of hypertension have persisted in the United Kingdom despite major investment in quality improvement initiatives, including pay for performance. These disparities are particularly marked among those patients with multiple cardiovascular conditions. For example, although the presence of 2 or more cardiovascular comorbidities was 


\section{Table 4. Odds of Antihypertensive Medication Prescription by Selected Characteristics}

\begin{tabular}{|c|c|c|c|c|c|}
\hline \multirow[b]{2}{*}{ Characteristic } & \multirow[b]{2}{*}{$\geq 3$ Medications } & \multicolumn{4}{|c|}{ Antihypertensive Medication } \\
\hline & & ACE Inhibitor & $\beta$-Blocker & Calcium Antagonist & Diuretic \\
\hline Age $^{\mathrm{a}}$ & $1.01(1.01-1.02)$ & $1.00(0.99-1.01)$ & $1.00(0.99-1.01)$ & $1.02(1.01-1.03)$ & $1.02(1.02-1.03)$ \\
\hline \multicolumn{6}{|l|}{ Ethnic group } \\
\hline White & 1.00 (ref) & 1.00 (ref) & 1.00 (ref) & 1.00 (ref) & 1.00 (ref) \\
\hline Black & $1.60(1.29-1.99)$ & $0.72(0.61-0.83)$ & $0.84(0.72-0.97)$ & $2.83(2.28-3.51)$ & $1.27(1.13-1.43)$ \\
\hline South Asian & $0.66(0.46-0.96)$ & $0.63(0.47-0.84)$ & $0.99(0.80-1.21)$ & $1.33(0.99-1.77)$ & $0.84(0.66-1.06)$ \\
\hline \multicolumn{6}{|c|}{ Number of comorbidities } \\
\hline 0 & 1.00 (ref) & 1.00 (ref) & 1.00 (ref) & 1.00 (ref) & 1.00 (ref) \\
\hline 1 & $2.31(1.87-2.85)$ & $2.55(2.19-2.96)$ & $1.46(1.21-1.75)$ & $1.28(1.11-1.48)$ & $1.03(0.93-1.15)$ \\
\hline$\geq 2$ & $4.99(2.45-6.54)$ & $3.25(2.59-4.06)$ & $2.33(1.89-2.87)$ & $1.51(1.24-1.83)$ & $2.03(1.72-2.40)$ \\
\hline
\end{tabular}

\section{Table 5. Number of Antihypertensive Medications Prescribed by Number of Cardiovascular Comorbidities and Ethnicity}

\begin{tabular}{lccc}
\hline $\begin{array}{l}\text { Medications } \\
\text { Prescribed }\end{array}$ & White, \%a & Black, \%a & South Asian, \%a \\
\hline 0 Comorbidity & & \\
0 Medications & $20.8(345 / 1,658)$ & $16.0(157 / 979)$ & $22.5(36 / 160)$ \\
1 Medication & $41.9(695 / 1,658)$ & $37.9(371 / 979)$ & $42.5(68 / 160)$ \\
2 Medications & $28.0(464 / 1,658)$ & $32.1(314 / 979)$ & $30.0(48 / 160)$ \\
$\geq 3$ Medications & $9.3(154 / 1,658)$ & $14.0(137 / 979)$ & $5.0(8 / 160)$ \\
1 Comorbidity & & & \\
0 Medications & $6.3(28 / 447)$ & $26.9(79 / 294)$ & $39.3(24 / 61)$ \\
1 Medication & $34.7(155 / 447)$ & $38.1(112 / 294)$ & $31.2(19 / 61)$ \\
2 Medications & $37.8(169 / 447)$ & $27.2(80 / 294)$ & $13.1(8 / 61)$ \\
$\geq 3$ Medications & $21.3(95 / 447)$ & & $0.0(0 / 28)$ \\
$\geq 2$ Comorbidities & & $1.5(1 / 66)$ & $21.4(6 / 28)$ \\
0 Medications & $4.4(4 / 90)$ & $37.9(25 / 66)$ & $50.0(14 / 28)$ \\
1 Medication & $18.9(17 / 90$ & $40.9(27 / 66)$ & $28.6(8 / 28)$ \\
2 Medications & $45.6(41 / 90)$ & & \\
$\geq 3$ Medications & $31.1(28 / 90)$ & & \\
\hline Note: Assessed only among patients with poorly controlled blood pressure (>140/90 mm Hg). & \\
a Values are expressed as percentage of patients (numerator/denominator). & \\
\hline
\end{tabular}

provide a comprehensive and typical picture of the care provided in this ethnically diverse, urban location. The high percentage of patients having their ethnicity coded in practice records $(94.8 \%)$ in this study is unique in a UK primary care setting. Combining what are known to be culturally and epidemiologically heterogeneous groups, that is, Indian, Pakistani, and Bangladeshi, into a South Asian group may have masked differences in associations seen between quality of care and number of comorbidities. Our sample may nonetheless have had insufficient power to detect an interaction effect between ethnicity, comorbidity, and mean diastolic blood pressure or achievement of the treatment target for hypertension.

Although our study provides an informative snapshot of current levels of care, use of cross-sectional data may introduce bias and does not permit us to determine whether there is a causal link between quality initiatives, such as pay for performance, and the variations in management of hypertension that we identified. More robust studies using longitudinal data with multiple measurement points and longer-term follow-up of patients are required to better evaluate the impact of these initiatives. We examined the relationship between cardiovascular comorbidities, rather than all comorbidities, and hypertension management in different ethnic groups. We cannot be sure that the associations found would apply to other common comorbidities, such as osteoarthritis or depression. The observed variations in 


\begin{tabular}{|c|c|c|c|}
\hline $\begin{array}{l}\text { Antihypertensive } \\
\text { Medication }\end{array}$ & White, \%a & Black, \%a & South Asian, \%a \\
\hline \multicolumn{4}{|l|}{0 Comorbidities } \\
\hline ACE inhibitor & $29.8(859 / 2,881)$ & $21.6(365 / 1,687)$ & $20.7(60 / 290$ \\
\hline$\beta$-Blocker & $23.1(665 / 2,881)$ & $18.9(318 / 1,687)$ & $22.8(66 / 290)$ \\
\hline Calcium antagonist & $30.8(888 / 2,881)$ & $52.9(892 / 1,687)$ & $35.2(102 / 290)$ \\
\hline Diuretic & $42.1(1,214 / 2,881)$ & $46.8(789 / 1,687)$ & $34.5(100 / 290)$ \\
\hline \multicolumn{4}{|l|}{1 Comorbidity } \\
\hline ACE inhibitor & $51.8(488 / 942)$ & $46.0(257 / 559)$ & $40.1(57 / 142)$ \\
\hline$\beta$-Blocker & $30.4(286 / 942)$ & $27.2(152 / 559)$ & $28.9(41 / 142)$ \\
\hline Calcium antagonist & $41.4(390 / 942)$ & $61.4(343 / 559)$ & $35.9(51 / 142)$ \\
\hline Diuretic & $46.5(438 / 942)$ & $47.6(266 / 559)$ & $40.1(57 / 142$ \\
\hline \multicolumn{4}{|l|}{$\geq 2$ Comorbidities } \\
\hline ACE inhibitor & $59.1(123 / 208)$ & $52.9(65 / 123)$ & $46.8(29 / 62)$ \\
\hline$\beta$-Blocker & $39.4(82 / 208)$ & $40.7(50 / 123)$ & $40.3(25 / 62)$ \\
\hline Calcium antagonist & $42.8(89 / 208)$ & $68.3(84 / 123)$ & $61.3(38 / 62)$ \\
\hline Diuretic & $65.4(136 / 208)$ & $67.5(83 / 123)$ & $50.0(31 / 62)$ \\
\hline
\end{tabular}

blood pressure control may partly reflect variations in adherence to medications, which have been shown to systematically differ between the groups we studied. ${ }^{16}$ Finally, given the major differences between the UK and US health care systems, the findings may not all be transferable to the United States. For example, the United Kingdom's National Health Service provides universal coverage with access to primary care services free at the point of use. As a result, patients from ethnic minority groups in the United Kingdom, who are more likely to come from lower socioeconomic groups or to be unemployed, do not face financial barriers in accessing primary health care services.

Some previous US studies published on this issue suggest that the provision of health care to individuals with multiple conditions is of a similar quality to or better than that delivered to individuals with a single condition. For example, a study of more than 6,000 individuals with diabetes enrolled in a managed care organization found that quality of care did not vary with the presence of medical comorbidity, as measured by the Charlson Comorbidity Index. ${ }^{17}$ Higashi et $\mathrm{al}^{18}$ found that quality of care actually improved with an increasing number of conditions in 3 cohorts of community-dwelling adult patients. Both studies examined quality using process of care indicators across a larger number of conditions than examined here, including both cardiovascular and noncardiovascular comorbidities. These findings may reflect the fact that patients with multiple conditions have greater contact with health services. The results of our study suggest that this quality gain may extend to better control of intermediate clinical outcomes, such as blood pressure, which is in keeping with previous research on blood pressure control among patients with multiple cardiovascular comorbidities. ${ }^{19}$ Our findings also suggest, however, that this improved control may be restricted to white patients with comorbidities and does not extend to black and South Asian patients.

The number of people living with multiple cardiovascular conditions globally is high and set to increase, ${ }^{20,21}$ particularly in countries with aging populations and rapidly rising levels of obesity. This group represents a high priority for secondary prevention efforts given their increased likelihood of additional morbidity and mortality, and associated use of health care resources. Our findings suggest that the management of hypertension in ethnic minority groups remains suboptimal, particularly in individuals with cardiovascular comorbiditiesdespite a sustained period of investment in health care quality improvement in England and a health care system that offers universal access to services free at the point of use.

Policy makers and health care planners should consider the potential negative impacts of universal quality improvement programs, such as pay for performance, on health care disparities. Although such programs generally aim to improve overall levels of care, our findings suggest that additional efforts are required to improve the quality of care among highrisk individuals, including those from minority ethnic groups with multiple conditions. Such improvement could be facilitated by, for example, offering additional incentives to meet treatment targets in people who are from ethnic minority groups or who have multiple morbidities.

To read or post commentaries in response to this article, see it online at http://www.annfammed.org/cgi/content/full/6/6/490.

Key words: Pay for performance; hypertension; disease management; blood pressure; ethnicity; cardiovascular diseases; comorbidities; primary care; practice-based research

Submitted February 18, 2008; submitted, revised, June 16, 2008; accepted June 24, 2008. 
Funding support: This study represents independent research commissioned by the National Institute for Health Research. The Wandsworth Primary Care Research Centre has received funding from the Department of Health. We are grateful for support from the National Institute for Health Research Biomedical Research Centre Funding Scheme, and the Medical Research Council.

Disclaimer: The views expressed in this publication are those of the authors and not necessarily those of the National Health Service, the National Institute for Health Research, or the Department of Health.

Acknowledgments: We thank the primary care practices that participated in the study. C.M. and J.G. conceived the study. C.M. and A.B. performed the statistical analyses. All authors contributed to the data interpretation. C.M. wrote the first draft of the manuscript, and all authors contributed to the revision and approved the final version. C.M is the guarantor for the study.

\section{References}

1. Chaturvedi N, McKeigue PM, Marmot MG. Resting and ambulatory blood pressure differences in Afro-Caribbeans and Europeans. Hypertension. 1993;22(1):90-96.

2. Sproston K, Mindell J. Health Survey for England 2004. Volume 1: The Health of Minority Ethnic Groups. 2004. The Information Centre. http:// www.ic.nhs.uk/statistics-and-data-collections/health-and-lifestylesrelated-surveys/health-survey-for-england. Accessed Jan 15, 2008.

3. Khattar RS, Swalesb JD, Seniora R, Lahiria A. Racial variation in cardiovascular morbidity and mortality in essential hypertension. Heart. 2000;83(3):267-271

4. Forouhi NG, Merrick D, Goyder E, et al. Diabetes prevalence in England, 2001-estimates from an epidemiological model. Diabet Med. 2006;23(2):189-197.

5. Lanting LC, Joung IMA, Mackenbach JP, Lamberts SWJ, Bootsma $\mathrm{AH}$. Ethnic differences in mortality, end-stage complications, and quality of care among diabetic patients: a review. Diabetes Care. 2005;28(9):2280-2288.

6. Leatherman S, Sutherland K. The Quest for Quality in the NHS A Mid-Term Evaluation of the Ten-Year Quality Agenda. London, England: The Nuffield Trust; 2003

7. Shekelle P. New contract for general practitioners. BMJ. 2003;326 (7387):457-458.

8. Millett C, Gray J, Saxena S, Netuveli G, Khunti K, Majeed A. Ethnic disparities in diabetes management and pay-for-performance in the UK: the Wandsworth Prospective Diabetes Study. PLoS Med. 2007;4(6):e191.
9. UK Department of Health. Health inequalities: progress and next steps. June 2008. http://www.dh.gov.uk/en/Publicationsandstatistics/Publications/PublicationsPolicyAndGuidance/DH_085307. Accessed Jun 16, 2008.

10. Brown AF, Ettner SL, Piette J, et al. Socioeconomic position and health among persons with diabetes mellitus: a conceptual framework and review of the literature. Epidemiol Rev. 2004;26:63-77.

11. Department of Communities and Local Government. Index of Multiple Deprivation. 2004. http://www.communities.gov.uk/archived/ general-content/communities/indicesofdeprivation/216309/. Accessed Jan 15, 2008.

12. Office for National Statistics. The census in England and Wales. April 2001. http://www.statistics.gov.uk/census. Accessed Jan 15, 2008.

13. UK National Health Service. Clinical Terms (the Read Codes) Version 3 Reference Manual. 2000. http://www.connectingforhealth.nhs.uk/terminology/readcodes/publications. Accessed Jan 24, 2008.

14. Huber PJ. The behavior of maximum likelihood estimates under nonstandard conditions. In: Proceedings of the Fifth Berkeley Symposium on Mathematical Statistics and Probability. Vol 1. Berkeley, CA: University of California Press; 1967:221-223.

15. Williams B, Poulter NR, Brown MJ, et al. The BHS Guidelines Working Party guidelines for management of hypertension: report of the fourth working party of the British Hypertension Society, 2004-BHS IV. J Hum Hypertens. 2004;18(3):139-185.

16. Morgan M. The significance of ethnicity for health promotion: patients' use of anti-hypertensive drugs in inner London. Int J Epidemiol. 1995;24(Suppl 1):S79-S84

17. Halanych JH, Safford MM, Keys WC, et al. Burden of comorbid medical conditions and quality of diabetes care. Diabetes Care. 2007;30(12):2999-3004.

18. Higashi T, Wenger NS, Adams JL, et al. Relationship between number of medical conditions and quality of care. $N$ Engl J Med. 2007;356(24):2496-2504

19. Wong ND, Lopez VA. L'Italien G, Chen R, Kline SE, Franklin SS. Inadequate control of hypertension in US adults with cardiovascular disease comorbidities in 2003-2004. Arch Intern Med. 2007;167(22):2431-2436

20. Fortin M, Bravo G, Hudon C, Vanasse A, Lapointe L. Prevalence of multimorbidity among adults seen in family practice. Ann Fam Med. 2005;3(3):223-228.

21. Wolff JL, Starfield B, Anderson G. Prevalence, expenditures, and complications of multiple chronic conditions in the elderly. Arch Intern Med. 2002;162(20):2269-2276. 